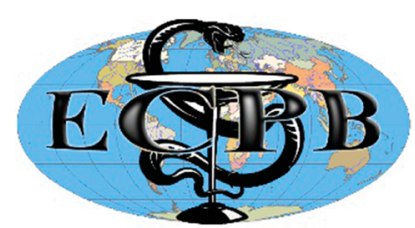

UDC 612.35:612.36:612.015.81
“ЕКСПЕРИМЕНТАЛЬНА ТА КЛІНІЧНА ФІЗІОЛОГІЯ І БІОХІМІЯ” "EXPERIMENTAL AND CLINICAL PHYSIOLOGY AND BIOCHEMISTRY" Науково-практичний журнал/Scientific-practical journal

Наукові статті / Research article ECPB 2019, 2(86): 24-30. https://doi.org/

\title{
The range of bile acid cholates in the bile of rats with steatohepatosis induced by a high-calorie diet
}

\author{
M.M. KONDRO ${ }^{1}$, S.P. VESELSKYI ${ }^{2}$, T.V. DOVBYNCHUK ${ }^{2}$, I.YU. PRYBYTKO ${ }^{2}$ \\ 1 Danylo Halytskyi Lviv National Medical University, Lviv, Ukraine \\ 2 Taras Shevchenko Kyiv National University, Kyiv, Ukraine
}

The effect of a high-calorie diet on the dynamics of choleresis and secretion of free and conjugated fatty acids was studied in experiments on rats. They has shown that in comparison with rats fed with the standard food, in rats that were on a high-calorie diet for 20 weeks, the volume rate of bile secretion increased by $12.4 \%(p<0.05)$. Using the method of thin layer chromatography, it has been found out that in the bile of rats within a 3-hour experiment the concentration of taurocholic acid mixture of taurochenodeoxycholic and taurodeoxycholic acids, the concentration of the mixture of glycochenodeoxycholic and glycodeoxycholic acids, the concentration of the mixture of chenodeoxycholic and deoxycholic acids increased. At the same time, the concentration of glycocholic acid in the bile of rats decreased after 20 weeks of rats' staying on HCD, the concentration of cholic acid did not undergo statistically significant changes. In this case, the conjugation coefficient remains unchanged and the hydroxylation coefficient has decreased which indicates the activation of some alternative pathway of biosynthesis of bile acids.

The incidence of nonalcoholic steatosis, also called as a nonalcoholic fatty liver disease, is growing rapidly around the world. The growth rate is $30-40 \%$, which is becoming a huge medical and economic burden for patients and states in the whole [1]. Therefore, the establishment of mechanisms of pathogenesis of steatohepatosis, its consequences and the development of optimal methods of treatment is an urgent problem of our time.

Previously, we have shown that the presence of rats on a high-calorie diet No. 11024 (HCD) (diet No. 11024, Research Diets, New Brunswick, NJ) for 20 weeks leads to the development of metabolic syndrome (MS), on the background of which liver steatosis was recorded $[2,3]$. On the background of structural changes in the liver, there is a change in its functional state, for the assessment of which it is important to determine the spectrum of bile acids in the bile and to establish a ratio between individual bile acids. Under physiological conditions, this spectrum is quite stable. However, in case of impairments of the hepatobiliary system, the content of individual bile acids can vary significantly.

Despite the fact that HCD No. 11024 is widely used in experimental studies, the aim of this work was to investigate the range of bile acids cholates in the bile of rats after 20 weeks of their staying on this diet.

Materials and methods. Studies were carried out on male white rats with an initial mass of 210-215 grams. During the first week all rats received the standard food and water ad libitum. On the 2nd week the animals were randomized into two groups. Rats of group 1 received the standard food and water ad libitum and served as a control group. Rats of group II were on the HCD (diet No. 11024, Research Diets, New Brunswick, NJ), which consisted 
of the standard feed (47\%), sweet concentrated milk (44\%), corn oil (8\%) and vegetable starch (1\%). This diet was chosen by us on the basis of the analysis of literature data on which the diet No. 11024 causes the development of steatohepatosis in rats [4] and mice [5].

After 20 weeks, rats were anesthetized with urethane (Sigma Aldrich, USA) $(1 \mathrm{~g} / \mathrm{kg}$, intraperitoneal). Then the common bile duct was cannulized in rats. Within 3 hours of observation, half-hour bile samples were taken. For unit that characterizes the secretory function of the liver we considered the average volume rate of secretion of bile, which was calculated based on the volume of bile (mcl) that was secreted for $1 \mathrm{~min}$ relative to 1 gram of liver [6].

Since the intensity of bile secretion and its properties determine bile acids $[7,8]$, the concentration of individual free and conjugated BA was determined in the collected half-hour bile samples using thin-layer chromatography and densitometer DO-1M in each bile sample [9]. With the help of 'Silufol' plates we found free hydrochloric acid and its individual Tauro-and glycoconjugates in the bile of rats (taurocholic acid and glycocholic acid). In the mixture of bile acids three fractions of deoxycholanic acids were detected: taurochenodeoxycholic and taurodeoxycholic, glycochenodeoxycholic and glycodeoxycholic, chenodeoxycholic and deoxycholic, respectively. The sensitivity of the method was $0.25-0.35 \mu \mathrm{g}$ of bile acid in the sample.

The important indicators that characterize the biochemical and physiological processes that occur during the secretion of bile are the coefficients of conjugation and hydroxylation. In a half-hour bile samples the ratio conjugation, the ratio of the concentrations of the conjugate (the sum of concentrations of taurocholic, taurochenodeoxycholic and taurodeoxycholic, glycocholic, glycochenodeoxycholic and glycodeoxycholic acids) to free bile acids (the sum of concentrations of cholic, chenodeoxycholic and deoxycholic acids), and the ratio of hydroxylation was determined by the ratio of the concentrations of trihydroxycholic (the sum of concentrations of taurocholic, glycocholic and cholic acids) to dihydroxycholic bile acids (the sum of concentrations of taurochenodeoxycholic and taurodeoxycholic, glycochenodeoxycholic and glycodeoxycholic, chenodeoxycholic and deoxycholic) were determined.

The statistical processing of the research results was carried out by conventional methods of variation statistics, according to which the obtained data were tested for the normality of the Shapiro-Wilk distribution. Since it was found that the results were normally distributed, the Student's t-test for independent samples was used for processing the statistical data.

Research results and discussion. The volume rate of bile secretion in rats of the control group was $1.21+0.03 \mu \mathrm{l} / \mathrm{g}$ liver $\bullet \min$. Rats that were on HCD for 20 weeks developed a severe visceral obesity, accompanied by steatohepatosis which was described by us in previous publications [2,3]. In rats on HCD, the volume rate of bile secretion increased to $1.36+0.03 \mu \mathrm{l} / \mathrm{g}$ of liver $\cdot \mathrm{min}$, or by $12.4 \%(p<0.05)$ which is obviously the result of the increased bile secretion liver function in response to HCD with high fat content.

In rats of the control group the concentration taurocholic acid and other bile acids for 3-hour experiment remained practically unchanged (Table 1). After 20 weeks on HCD the concentration of taurocholic acid in rats grew in all samples (Table. 1$)$. In the first sample it grew by $10.2 \%(p<0.05)$ and in the sixth - by $15.3 \%(p<0.05)$ (Table 1$)$.

The concentration of the compound taurochenodeoxycholic and taurodeoxycholic acids in rats after 20 weeks of rats on HCD grew to an even greater extent in all samples of the experiment. The lowest growth was recorded in the first sample $(30.3 \%, p<0.05)$, the highest - in the sixth $(36.3 \%, p<0.05)$ (Table 1). It is known that taurocholic bile acids have a choleretic effect and thereby prevent the cholestasis unlike glycine conjugated bile acids [10]. Indeed, in our experiments in rats after 20 weeks on HCD, the volume rate of bile secretion increased. 
Unlike tauroholic acid, the concentration of glycocholic acid in the bile of rats after 20 weeks of rats' staying on the HCD decreased during all periods of observation. At the beginning of the experiment (the first 30-minute sample) it decreased by $35.3 \%(p<0.05)$ and at the end of the experiment (the sixth 30 -minute sample) - by $43.9 \%(p<0.05)$ (Table 1$)$.

Table 1

Changes in the concentration of bile acids in the bile of rats caused by staying on a high-calorie diet for 20 weeks $(M+m, n=6)$

\begin{tabular}{|c|c|c|c|}
\hline \multirow{2}{*}{ Bile acids } & \multirow{2}{*}{$\begin{array}{l}\text { Sample } \\
\text { number }\end{array}$} & \multicolumn{2}{|c|}{ Bile acid concentration in $\mathrm{mg} \%$} \\
\hline & & control & High calorie diet \\
\hline \multirow{6}{*}{ Taurocholic acid } & 1 & $197.2 \pm 6.4$ & $217.4 \pm 4.1^{*}$ \\
\hline & 2 & $199.3 \pm 5.8$ & $226.1 \pm 2.6^{*}$ \\
\hline & 3 & $202.7 \pm 6.1$ & $226.7 \pm 4.2^{*}$ \\
\hline & 4 & $197.5 \pm 4.6$ & $221.9 \pm 4.2^{*}$ \\
\hline & 5 & $190.9 \pm 5.1$ & $220.3 \pm 3.2^{*}$ \\
\hline & 6 & $186.8 \pm 5.0$ & $215.4 \pm 3.2^{*}$ \\
\hline \multirow{6}{*}{$\begin{array}{l}\text { The mixture of } \\
\text { taurochenodeoxycholic and } \\
\text { taurodeoxycholic }\end{array}$} & 1 & $104.2 \pm 6.3$ & $135.8 \pm 4.6^{*}$ \\
\hline & 2 & $107.3 \pm 8.5$ & $142.6 \pm 5.5^{*}$ \\
\hline & 3 & $107.4 \pm 7.6$ & $143.3 \pm 5.0^{*}$ \\
\hline & 4 & $101.9 \pm 7.5$ & $134.9 \pm 4.7^{*}$ \\
\hline & 5 & $97.2 \pm 6.0$ & $132.0 \pm 4.0^{*}$ \\
\hline & 6 & $92.6 \pm 6.3$ & $126.2 \pm 3.0^{*}$ \\
\hline \multirow{6}{*}{ Glycocholic acid } & 1 & $149.0 \pm 12.8$ & $96.4 \pm 7.7^{*}$ \\
\hline & 2 & $149.8 \pm 10.9$ & $95.8 \pm 8.1^{*}$ \\
\hline & 3 & $152.6 \pm 9.6$ & $90.7 \pm 7.8^{*}$ \\
\hline & 4 & $142.7 \pm 12.0$ & $83.8 \pm 6.0^{*}$ \\
\hline & 5 & $139.4 \pm 11.9$ & $78.4 \pm 5.2^{*}$ \\
\hline & 6 & $131.3 \pm 12.3$ & $73.7 \pm 5.1^{*}$ \\
\hline \multirow{6}{*}{$\begin{array}{l}\text { The mixture of } \\
\text { glycochenodeoxycholic and } \\
\text { glycodeoxycholic acids }\end{array}$} & 1 & $34.5 \pm 3.0$ & $46.5 \pm 4.3^{*}$ \\
\hline & 2 & $35.5 \pm 2.9$ & $47.3 \pm 4.6^{*}$ \\
\hline & 3 & $35.1 \pm 2.5$ & $45.8 \pm 4.1^{*}$ \\
\hline & 4 & $32.9 \pm 3.1$ & $43.8 \pm 4.4^{*}$ \\
\hline & 5 & $30.9 \pm 3.0$ & $43.9 \pm 4.4^{*}$ \\
\hline & 6 & $29.3 \pm 2.9$ & $41.5 \pm 4.4^{*}$ \\
\hline \multirow{6}{*}{ Holic acid } & 1 & $25.0 \pm 1.4$ & $21.5 \pm 1.1$ \\
\hline & 2 & $24.9 \pm 0.7$ & $21.8 \pm 1.2$ \\
\hline & 3 & $23.8 \pm 0.9$ & $22.1 \pm 1.1$ \\
\hline & 4 & $23.2 \pm 1.2$ & $21.7 \pm 1.1$ \\
\hline & 5 & $22.2 \pm 1.8$ & $21.6 \pm 1.4$ \\
\hline & 6 & $22.4 \pm 2.1$ & $21.0 \pm 1.8$ \\
\hline \multirow{6}{*}{$\begin{array}{l}\text { The mixture of } \\
\text { chenodeoxycholic and } \\
\text { deoxycholic }\end{array}$} & 1 & $12.5 \pm 0.5$ & $15.5 \pm 0.8^{*}$ \\
\hline & 2 & $12.4 \pm 0.5$ & $16.8 \pm 0.6^{*}$ \\
\hline & 3 & $13.2 \pm 0.9$ & $17.4 \pm 0.4^{*}$ \\
\hline & 4 & $13.1 \pm 0.9$ & $16.5 \pm 0.8^{*}$ \\
\hline & 5 & $13.6 \pm 0.9$ & $15.6 \pm 1.0$ \\
\hline & 6 & $13.1 \pm 1.1$ & $16.4 \pm 0.6^{*}$ \\
\hline
\end{tabular}

Note: $*-p<0.05$ as compared to the control group; HCD - a high-calorie diet. 
On the contrary, the concentration of the mixture of glycochenodeoxycholic and glycodeoxycholic increased in all samples. In the first sample it grew by $34.8 \%(p<0.05)$, and in the sixth - by $41.6 \%(p<0.05)$ (Table 1$)$.

The concentration of cholic acid in the bile of rats, which were 20 weeks on HCD, did not undergo statistically significant changes (Table 1).

The concentration of the mixture of chenodeoxycholic and deoxycholic increased in all samples. Unlike taurocholic acid mixture of glycochenodeoxycholic and glycodeoxycholic acids, mixtures of glycochenodeoxycholic and glycodeoxycholic acids, the concentration of which did not undergo significant fluctuations during the experiment, the concentration of the mixture of chenodeoxycholic and deoxycholic acids in dynamics experienced the following changes: in the first, second, third, fourth and sixth trial, it has grown by $24.0 \%(p<0.05)$ and by $35.5 \%(p<0.05)$ and by $31.8 \%(p<0.05)$, by $26.0 \%$ $(p<0.05)$ and $25.2 \%(p<0.05)$ respectively. In the fifth sample, the increase in the concentration of the mixture of chenodeoxycholic and deoxycholic acids was statistically unreliable.

According to the literature referring to the bile of rats cholic, chenodeoxycholic, deoxycholic, lithocholic acids and their conjugates with taurine and glycine are characteristic. The first quantitative content in the bile of rats is the concentration of taurocholic acid - cholic acid conjugated with taurine. The second quantitative content of bile is glycocholic acid. The data obtained by us showed their compliance with the existing data in the literature, the rats of the control group had the highest concentration of taurocholic acid (from $186.8 \pm 5.0 \mathrm{mg} \%$ to $202.7 \pm 6.1 \mathrm{mg} \%$ ). Then came the concentration of glycocholic acid (from of $131.3 \pm 12.3 \mathrm{mg} \%$ to $152.6 \pm 9.6 \mathrm{mg} \%)$. The third quantitative content in the bile of rats was the concentration of the sum of taurochenodeoxycholate and taurodeoxycholate acids $(92.6 \pm 6.3 \mathrm{mg} \%$ to $107.4 \pm 7.6 \mathrm{mg} \%)$ which also corresponds to the data of other authors [11]. Much less than in conjugated bile acids the concentration in the bile of rats of free bile acids - trihydroxycholanic cholic (from $22.4 \pm 2.1 \mathrm{mg} \%$ to $25.0 \pm 1.4 \mathrm{mg} \%$ ) and dihydroxycholanic and deoxycholanic (from $12.4 \pm 0.5 \mathrm{mg} \%$ to $13.6 \pm 0.9 \mathrm{mg} \%$ ).

Our results indicate that the long staying of rats on HCD results in the development of steatohepatosis, increases choleresis and significantly changes the spectrum of cholates in bile. On the background of the unchanged concentration of free bile acid - cholic, increases the concentration of free chenodeoxycholic and deoxycholic acids. It has been observed that after 20 weeks of staying on HCD the concentration of taurocholic acid in the bile of rats has increased, which may be the evidence of activation of multienzyme systems of cholic acid conjugation with taurine, and the result of increased biosynthesis and transport taurocholate through the plasma membrane of hepatocytes. In this case, conjugation of cholic acid with glycine in hepatocytes was inhibited, which was manifested in the decrease in the concentration of glycocholic acid in the bile of experimental animals. It is worth noting that the processes of conjugating cholic acid with glycine were significantly inhibited in male hepatocytes. However, the calculation of the coefficient of conjugation of bile acids in the bile of rats after a long stay on the HCD showed that it did not undergo statistically significant changes, except for the 5th sample, in which it grew by $15.3 \%(p<0.05)$ (Table 2$)$.

As it can be seen from table 3 , the rate of hydroxylation of bile acids in the bile of rats of the control group did not undergo significant fluctuations and amounted from 2.4 to 2.5 , which is consistent with the existing data in the literature, in which the coefficient of hydroxylation of bile acids in intact rats bile of the corresponding age ranged from 2.3 to 2.7 [12] and from 2.21 to 2.51 [11].

In contrast to the conjugation coefficient, the coefficient of hydroxylation of bile acids in the bile of rats, which were on the HCD for a long period of time, decreased in the 1st, 4th, 5th and 6th samples by $32.0 \%(p<0.05)$, in the 2 nd - by $29.2 \%(p<0.05)$ and in the $3 r d-$ by $33.3 \%(p<0.05)$. 
Table 2

Changes in the conjugation coefficient and the concentration of bile acids in the bile of rats caused by staying on a high-calorie diet for 20 weeks $(M+m, n=6)$

\begin{tabular}{|c|c|c|c|}
\hline $\begin{array}{c}\text { Sample } \\
\text { No. }\end{array}$ & $\begin{array}{c}\text { Amount of conjugated bile } \\
\text { acids, } \mathbf{~ m g} \%\end{array}$ & $\begin{array}{c}\text { The amount of free bile } \\
\text { acids, } \mathbf{~ m g} \%\end{array}$ & Conjugation ratio \\
\hline \multicolumn{5}{|c|}{ The control group } \\
\hline 1 & $484.9 \pm 7.1$ & $37.5 \pm 1.0$ & 12.9 \\
\hline 2 & $490.9 \pm 7.0$ & $37.3 \pm 0.6$ & 13.2 \\
\hline 3 & $497.8 \pm 6.5$ & $37.0 \pm 0.9$ & 13.4 \\
\hline 4 & $475.0 \pm 6.8$ & $36.3 \pm 1.1$ & 13.1 \\
\hline 5 & $398.4 \pm 6.5$ & $35.8 \pm 1.4$ & 11.1 \\
\hline 6 & $440.0 \pm 6.6$ & $35.5 \pm 1.6$ & 12.4 \\
\hline \multicolumn{5}{|c|}{ The group of rats on a high-calorie diet } \\
\hline 1 & $496.1 \pm 5.2$ & $37.0 \pm 1.0$ & 13.4 \\
\hline 2 & $511.8 \pm 5.2$ & $38.6 \pm 0.9$ & 13.2 \\
\hline 3 & $506.5 \pm 5.3$ & $39.5 \pm 0.8$ & 12.8 \\
\hline 4 & $484.4 \pm 4.8$ & $38.2 \pm 1.0$ & 12.7 \\
\hline 5 & $474.6 \pm 4.2^{*}$ & $37.2 \pm 1.2$ & 12.8 \\
\hline 6 & $456.8 \pm 3.9$ & $37.4 \pm 1.2$ & 12.2 \\
\hline
\end{tabular}

Note: $*-p<0.05$ compared to the corresponding test in the control.

This result is due to the change in the ratio of the concentrations of trihydroxycholanic to dihydroxycholanic bile acids: during the reduction in the concentration of trihydroxycholanic bile acids, the concentration of dihydroxycholanic bile acids has increased steadily in all samples.

There are two ways of synthesis of bile acids. The first is classical or neutral, induced by cholesterol-7a-hydroxylase (CYP7A1) [13]. The result of the activation of this pathway is the synthesis of cholic acid. The second path is the alternative or acidic one. It is stimulated by CYP27A1 and leads to the generation of chenodeoxycholic acid. As in our experiments in rats after 20 weeks of staying on HCD the concentration of cholic acid in the bile of rats has not changed, and the concentration of deoxycholic and chenodeoxycholic has grown, we came to the conclusion about the strengthening of their biosynthesis by the 'acid' way with the involvement of the mitochondrial enzymes (Table 3).

Table 3

Changes in the coefficient of hydroxylation and the concentration of bile acids in the bile of rats caused by staying on a high-calorie diet for 20 weeks $(M+m, n=6)$

\begin{tabular}{|c|c|c|c|}
\hline $\begin{array}{c}\text { Sample } \\
\text { No. }\end{array}$ & $\begin{array}{c}\text { Trihydroxycholanic } \\
\text { bile acids }\end{array}$ & $\begin{array}{c}\text { Dihydroxycholanic } \\
\text { bile acids }\end{array}$ & $\begin{array}{c}\text { The ratio of } \\
\text { hydroxylation }\end{array}$ \\
\hline \multicolumn{4}{|c|}{ The control group } \\
\hline 1 & $371.2+6.9$ & $151.2+3.3$ & 2.5 \\
\hline 2 & $374.0+5.8$ & $155.2+4.0$ & 2.4 \\
\hline 3 & $377.4+5.5$ & $155.7+3.6$ & 2.4 \\
\hline 4 & $363.4+5.9$ & $147.9+3.8$ & 2.5 \\
\hline 5 & $352.5+6.3$ & $141.7+3.3$ & 2.5 \\
\hline 6 & $340.5+6.5$ & $135.0+3.4$ & 2.5 \\
\hline
\end{tabular}




\begin{tabular}{|l|l|l|l|}
\hline \multicolumn{4}{|c|}{ The group of rats on a high-calorie diet } \\
\hline 1 & $335.3+4.3^{*}$ & $197.8^{*}+3.2^{*}$ & 1.7 \\
\hline 2 & $343.7+4.0^{*}$ & $206.7+3.6^{*}$ & 1.7 \\
\hline 3 & $339.5+4.4^{*}$ & $206.5+3.2^{*}$ & 1.6 \\
\hline 4 & $327.4+3.8^{*}$ & $195.2+3.3^{*}$ & 1.7 \\
\hline 5 & $323.1+3.3^{*}$ & $191.5+3.2^{*}$ & 1.7 \\
\hline 6 & $310.1+3.4^{*}$ & $184.1+2.6^{*}$ & 1.7 \\
\hline
\end{tabular}

Note: $*-p<0.05$ compared to the corresponding test in the control.

Conclusions. Thus, a long staying of rats on a high-calorie diet, has resulted in the development of steatohepatosis, increased choleresis and significantly changed the composition of the bile of rats. After 20 weeks on a high calorie diet in the bile of rats has increased the concentration of bile acids tauroconjugates, which obviously is the basis of bile secretion volume strengthening mechanism. In this case, the conjugation coefficient has not changed, and the hydroxylation coefficient decreased, which indicates the activation of the alternative pathway of biosynthesis of bile acids.

\section{Рекомендовано до друку колісією з біоетики}

\section{REFERENCES}

1. Younossi ZM, Koenig AB, Abdelatif D, Fazel Y, Henry L, Wymer M. Global epidemiology of 9on-alcoholic fatty liver disease - metaanalytic assessment of prevalence, incidence and outcomes. Hepatology. 2016;64:73-84.

2. Bodnar PM, Dinnik OB, Michalchyshyn GP, Kondro MM, Beregova TV. Estimation of shear wave elastography in the diagnosis of experimental non-alcoholic fatty liver disease. Journal of NAMS of Ukraine. 2011;17(4):422-30.

3. Bodnar PM, Dinnick OB, Mikhalchishin GA, Beregova TV, Kobilyak NM, Falaleeva TM, Kondro MM. Diagnostic efficiency of the new ultrasound method for assessing the stiffness of the liver - elastration of the shear wave in animals with experimental obesity. Reports of the National Academy of Sciences of Ukraine. 2013;4:159-66.

4. West DB, Boozer CN, Moody DL, Atkinson RL. Dietary obesity in nine inbred mouse strains. American Journal of Physiology. 1992 June;262(6 Pt 2):R1025-32.

5. Chanadyry $D$. Correction of oxidative stress in immunocompetent cells (in splenocytes) under the action of a complex of catechins of green tea on the model of experimental alimentary obesity. Author's abstract...andidate medical sciences. 14.00.36. Allergology and Immunology. Tbilisi. 2006. 20 p.

6. Kartifuzova ZhV, Reshetnik YM, Bondzik OV, Veselskyi SP, Makarchuk MY. Changes in the bile acid composition of the bile under the influence of ethanol under the blockade of opioid receptors by naloxone. Physics of the Living. 2008;16(12):116-21.

7. Veselskyi SP, Makarchuk My, Yanchuk PI. Features of the spectrum of bile acids in humans and animals. Scientific Visnyk. Volyn State University named after Lesia Ukrainka. 2007;5:65-72.

8. Bondzyk OV, Reshetnik YeM, Veselskyi SP, Yanchuk PI. Investigation of the effect of L-arginine on the bile duct liver function of rats. Scientific Visnyk. Volyn National University named after Lesia Ukrainka. SECTION 1. Physiology of humans and animals. 2010;12:9-14.

9. Veselskyi SP, Lyashchenko PS, Lukyanenko IA (USSR); A.S. 1624322 USSR, MBI G

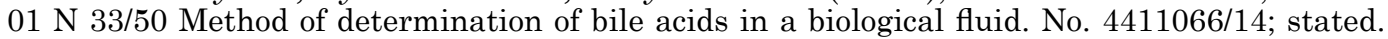
25.01.1988; Published on January 30, 1991, Bul. No 4.

10. $\mathrm{LiT}$, Chiang JY. Bile acid signaling in metabolic disease and drug therapy. Pharmacoly Review. 2014 Oct; 66 (4): 948-83. URL: doi: 10.1124/pr.113.008201.

11. Danchenko NM, Veselskyi SP, Tsudzevich BO. Ratio of bile acids in rats bile under conditions of development of aloxane-induced diabetes. Ukrainian Biochemical Journal. 2014;86(6):147-53.

12. Mozheitova OA, Nuryshchenko NE, Gorenko ZA, Karbovskaya LS, Lyaschenko TP. Characteristics of the ratio in the bile of dogs and rats in the use of prostaglandin F2a. Physics of living. 2009;17(2):179-84.

13. Zhua Y, Liub H, Zhangc M, Guo GL. Fatty liver diseases, bile acids, and FXR. Acta Pharmaceutica Sinica. B 2016;6(5):409-12. 


\title{
RESEARCH ARTICLE
}

\section{The Range of Bile Acid Cholates in the Bile of Rats With Steatohepatosis Induced by a High-calorie Diet}

\author{
M.M. KONDRO ${ }^{1}$, S.P. VESELSKYI ${ }^{2}$, T.V. DOVBYNCHUK ${ }^{2}$, I.YU. PRYBYTKO ${ }^{2}$ \\ ${ }^{1}$ Danylo Halytsky Lviv National Medical University, Lviv, Uktaine \\ ${ }^{2}$ Taras Shevchenko Kyiv National University, Kyiv, Uktaine
}

Inroduction. Previously, it has been demonstrated on rats that 20 week high-energy diet (HED) leads to the development of metabolic syndrome (MS) in the course of which the hepatic steatosis was observed. Taking into consideration the fact that the main function of bile acids (BA) is digestion and lipid absorption, the regulation of the cholesterol synthesis as well as the bile formation and secretion, the purpose of the article is to investigate the spectrum of cholates in BA in the bile of rats with MS.

Materials and methods. The research was conducted on female rats with initial weight of 210-215 grams. During the first week of the research all rats received the standard food and water ad libitum. During the 2 nd week the rats were divided into two groups at random: I - received the standard food and water ad libitum (the control group), II - was on HED (diet No. 11024, Research Diets, New Brunswick, NJ). Within 20 weeks the common bile duct was cannulated under urethane anesthesia ( $1 \mathrm{~g} / \mathrm{kg}$ of the body weight). During 3 hours of the observation the bile samples were selected every $30 \mathrm{~min}$. Using thin layer chromatography and densitometer DO-1M every sample of the bile was examined for the concentration of separate free or conjugated bile acids. On "Silufol" plates the bile acids were distributed as follows: taurocholic acid (TCA), the mixture of taurochenodeoxycholic and taurodeoxycholic acids (TCDCA+TDCA), glycocholic acid (GCA), the mixture of glycochenodeoxycholic and glycodeoxycholic acids (GCDA+GDCA), cholic acid (CA), the mixture of chenodeoxycholic and deoxycholic acids (CDA+DA).

Results. During the three-hour testing the concentration of TCA in rats' bile with MS increased in all 30-minute samples. In the 1st sample, the TCA concentration increase comprised $10.2 \%(p<0.05)$, in the 6th sample $-15.3 \%(p<0.05)$. Moreover, the overall level of TCDCA+TDCA was higher and increased by $30.3 \%(p<0.05)$ in the 1 st sample and by $36.3 \%$ $(p<0.05)$ in the 6th sample. Unlike tauroconjugated BA, the concentration of glycoconjugated BA in rats' bile with MS was changing in different directions. The concentration of GCA decreased by $35.3-43.9 \%(p<0.05)$ and the level of glycochenodeoxycholic GCDA+GDCA in all samples increased by $34.8-41.6 \%(p<0.05)$. The concentration of CA in the rats' bile with MS did not change significantly, thus the overall level of the free BA CDA+DA remained higher during the whole process of testing. The highest increase was in the second sample and made $35.5 \%(p<0.05)$ as compared with the control group.

Conclusions. Thus, the long staying of rats on a high-calorie diet resulted in the development of steatohepatosis, the increase of choleresis and significant changes in the composition of the bile of rats. Thus, under the conditions of MS development, the processes of trihydroxycholestenoic initial bile acid conjugation in hepatocytes were stimulated - cholic acid with taurine, while the processes of cholic acid conjugation with glycine were suppressed. The observed increase of TCA concentration in the rats' bile with MS may indicate the activation of intracellular enzymes which provide the conjugation of BA with taurine and is the result of the intensification of taurocholate transportation through hepatocytes cell membrane. In this case the conjugation coefficient was not changed and the hydroxylation coefficient decreased which indicates the activation of the alternative pathway of biosynthesis of bile acids.

Key words: high-calorie diet, bile secretion, bile acids, metabolic syndrome, non-alcoholic fatty liver disease. 